\title{
Annealing Effects on the Structural Properties of FIB Prepared Cu Nanopillars - an in situ TEM study
}

Daniel Kiener ${ }^{1}$, Zaoli Zhang ${ }^{2}$, Saso Šturm ${ }^{3}$, Sophie Cazottes ${ }^{2}$, Peter J. Imrich ${ }^{2}$, Christoph Kirchlechner ${ }^{1}$ and Gerhard Dehm ${ }^{1,2}$

1. Department Materials Physics, Montanuniversität Leoben, Leoben, Austria.

2. Erich Schmid Institute for Materials Science, Austrian Academy of Sciences, Leoben, Austria.

3. Institute Jožef Stefan, Ljubljana, Slovenia.

The unexpected mechanical size effects when reducing single crystal samples down to several $\mu \mathrm{m}$ dimensions or below has attracted considerable interest over the last couple of years [1, 2]. Studies of this kind became widely possible by the availability of focused ion beam microscopes (FIB) used for sample fabrication [3, 4]. The effect of FIB fabrication on the mechanical properties of miniaturized mechanical tests has recently been realized [5-7], but is not well documented. In this study, the effect of post thermal annealing on the plastic properties of FIB fabricated micro and nanometer sized $\mathrm{Cu}$ samples was studied by means of advanced analytic and in situ transmission electron microscopy (TEM).

Initially, the dislocation density of the investigated copper bulk single crystal was characterized to be $\sim 2.3 \cdot 10^{13} \mathrm{~m}^{-2}$. Then, selected areas of thin foils were intentionally FIB damaged, leading to a high defect density on the order of $1 \cdot 10^{15} \mathrm{~m}^{-2}$. In situ heating experiments of these thin films reveal a thermally driven reduction of the initially high dislocation density from FIB fabrication, but never recover the bulk dislocation density (Figure 1). The same holds true for nanoscale compression samples that were FIB prepared and subsequently in situ heated before mechanical testing (Figure 2a). Aberration corrected atomic imaging of the nano-compression samples documented the structural defects created during FIB milling, and also the recovery of a pristine crystalline surface structure upon annealing. Moreover, electron energy-loss spectroscopy (EELS) confirmed that the remaining contamination layer on the sample surface consisted of amorphous carbon, a typical contamination in electron microscopy.

These structural observations before and after annealing are combined with mechanical data from in situ tests of annealed micro- and nanometer sized tensile and compression samples. The micro-samples were loaded in situ in the scanning electron microscope, as described in [8], after annealing them in a vacuum furnace. The nano-compression specimens were, after annealing in the TEM, also loaded in situ in the TEM using a Hysitron PI-95 Picoindenter operated in displacement control, for experimental details see [9] (Figure 2).

For micron scale samples, the thermal annealing mainly influences the initial yield point, as it reduces the number of suited dislocation sources available in the specimen, while the flow behavior is mostly unaffected. For the submicron samples, the annealed material sustains significantly higher stresses throughout the whole deformation compared to FIB prepared specimens without heat treatment. This is explained by the high stresses required for surface mediated dislocation nucleation for the annealed material at the nanoscale. In the present case, the FIB affected surface near defects facilitate dislocation nucleation in copper, thereby lowering the material strength [10]. 
References:

[1] MD Uchic, DM Dimiduk, JN Florando, WD Nix, Science 305 (2004), p. 986.

[2] MD Uchic, PA Shade, DM Dimiduk, Ann. Rev. Mater. Res. 39 (2009), p. 361.

[3] MW Phaneuf, Micron 30 (1999), p. 277.

[4] CA Volkert, AM Minor, MRS Bull. 32 (2007), p. 389.

[5] H Bei, S Shim, MK Miller, GM Pharr, EP George, App. Phys. Lett. 91 (2007), p. 111915.

[6] D Kiener, C Motz, M Rester, G Dehm, Mater. Sci. Eng. A 459 (2007), p. 262.

[7] JA El-Awady, C Woodward, DM Dimiduk, NM Ghoniem, Phys. Rev. B 80 (2009), p. 104104.

[8] D Kiener, W Grosinger, G Dehm, R Pippan, Acta Mater. 56 (2008), p. 580.

[9] D Kiener, AM Minor, Acta Mater. 59 (2011), p. 1328.

[10] D Kiener, Z Zhang, S Šturm, S Cazottes, PJ Imrich, C Kirchlechner, G Dehm, Phil. Mag. 92 (2012), p. 3269.

[11] Support during sample preparation by G. Moser and H. Felber is gratefully acknowledged, and J. Thomas is thanked for TEM support,

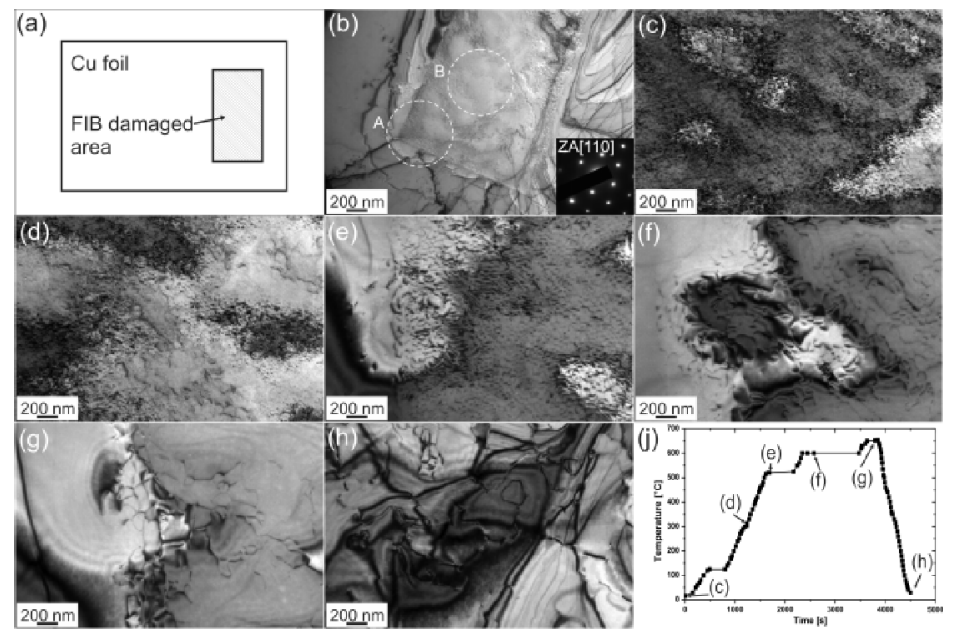

Figure 1. (a) Schematic of the heating sample. (b) Low magnification TEM image of a FIB damaged area in a (111) oriented $\mathrm{Cu}$ single crystal before in situ heating. Circles indicate the inspected areas in the corner (A) and center (B) of the FIB exposed material. The inset shows a diffraction pattern taken along the [110] zone axis. (c) Dislocation structure in area $\mathrm{B}$ at room temperature. (d-g) Dislocation arrangement in area A during heating at $300^{\circ} \mathrm{C}, 520^{\circ} \mathrm{C}, 600^{\circ} \mathrm{C}$, and $650^{\circ} \mathrm{C}$, respectively. (h) Dislocation structure in area $\mathrm{B}$ after cooling to room temperature. (j) Temperature profile with indication of the in situ images (b-h).
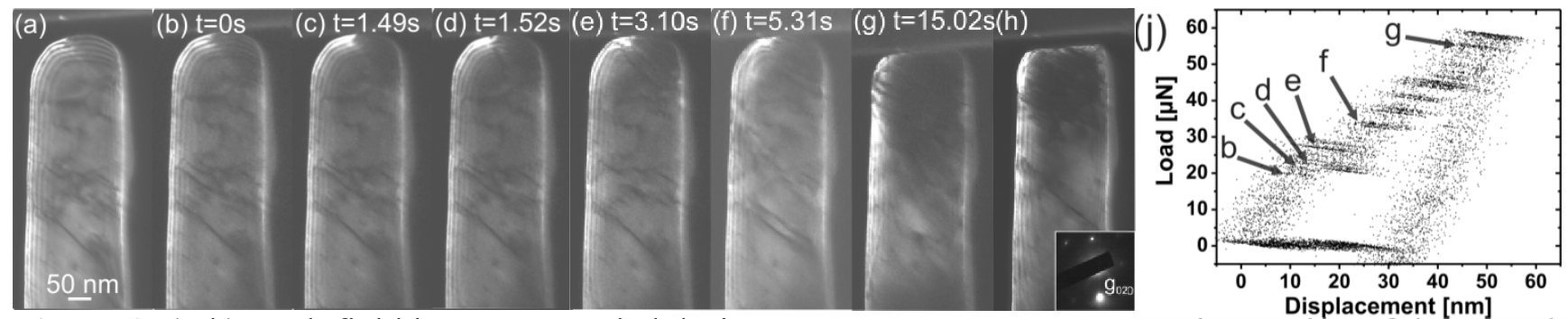

Figure 2. (a-h) Dark field images recorded during in situ TEM nano-compression testing of the annealed pillar using a $\mathrm{g}_{020}$ imaging condition. The time elapsed since first contact of sample and flat diamond punch is stated at the top of each image. (j) Load-displacement data correlated to the in situ images. 